\title{
Design Cibersemiótico: uma proposta metodológica para o ensino de Processos Digitais de Projeto
}

\author{
Cybersemiotic Design: a methodological purpose for digital design teaching \\ - Gilfranco Alves \\ algo+ritmo - Universidade Federal de Mato \\ Grosso do Sul, Brazil. \\ gilfranco.alves@ufms.br \\ - Juliana Trujillo \\ algo+ritmo - Universidade Federal de Mato \\ Grosso do Sul, Brazil. \\ juliana.trujillo@ufms.br
}

\begin{abstract}
The paper aims to relate a specific aspect of the proposed methodology for digital design, presented on the PhD research called Cibersemiótica e Processos de Projeto: Metodologia em Revisão, developed at the University of São Paulo (USP), with the didactic experience conducted at the subject Representação e Criação Digital 2, developed at the Architecture and Urbanism Course of the Federal University of Campo Grande (UFMS), Brazil. The paper's theoretical foundation is based on the cybersemiotic work produced by the Danish philosopher Søren Brier, which unifies two important conceptual frameworks: the Charles Sanders Peirce's Semiotics, and the Second Order Cybernetics proposed by Heinz von Foerster.
\end{abstract}

Keywords: Education, Design Processes, Parametric Design, Cybersemiotics

\section{Introdução}

A evolução dos meios digitais tem proporcionado significativas mudanças socioculturais, em função das modificações que ocorrem nos modos de vida contemporâneos. A velocidade e a dinâmica de disponibilização do conhecimento, as bases tecnológicas que se diversificam e se especializam cada vez mais, assim como as relações interdisciplinares e as trocas possíveis de serem feitas em níveis cada vez mais ampliados, vêm alterando o modo de se pensar, de se projetar e de se produzir em arquitetura e urbanismo.

Concomitantemente a esta gama de atualizações e desenvolvimentos, os processos digitais de projeto apresentam uma série de caminhos que se abrem, tanto de um ponto de vista teórico, discutindo e propondo abordagens que apontem outras possibilidades para problemas arquitetônicos contemporâneos, como de um ponto de vista prático, utilizando software, máquinas e equipamentos para otimizar a capacidade de produção e potencializar a performance da arquitetura que se pode produzir atualmente.

Se por um lado se torna importante o desenvolvimento teórico, na medida em que a proposição de estruturas, métodos ou esquemas conceituais traz outros olhares para a questão dos processos digitais de projeto, por outro, a evolução tecnológica possibilita a utilização de uma lógica que explora mais profundamente outros procedimentos construtivos e materiais que passam a ser utilizados, em função de suas propriedades físicas, químicas e biológicas.

Um possível discurso integrador e não-determinista em direção à construção de fundamentações que forneçam suporte para uma arquitetura produzida a partir da mediação digital, e que se imagine reunindo diferentes estratégias e as mais variadas pesquisas sobre processos digitais de projeto, necessita se concentrar, em nosso ponto de vista, tanto em aspectos de natureza teórica, dos mais diversos campos do conhecimento, quantoemaspectos denatureza prática, da ordem da experiência. Deste modo, acreditamos que o ensino de projeto em arquitetura e urbanismo precisa acompanhar as atualizações necessárias, incorporando as características dos modos de concepção a partir da programação, do uso de algoritmos e definições de parâmetros, e de sistemas de modelagem organizadores da informação (BIM). Estas possibilidades presentes nos processos digitais de projeto visam pesquisar outras abordagens dentro de uma diversidade de abordagens metodológicas possíveis de serem aplicadas, testadas, integradas e implementadas nos currículos e nas práticas do ensino de projeto nos cursos de arquitetura e urbanismo, especialmente no Brasil.

\section{Procedimentos metodológicos}

Este artigo pretende discutir a relação entre teoria e prática, partindo da pesquisa de doutorado intitulada "Cibersemiótica e Processos de Projeto: Metodologia em Revisão", defendida em 2014 junto à Universidade de São 
Paulo (USP) e uma experiência didática realizada no âmbito da disciplina "Representação e Criação Digital II", implementada no curso de arquitetura e urbanismo da Universidade Federal de Mato Grosso do Sul (UFMS) a partir de 2014.

Também auxiliam e contextualizam a discussão outras duas oficinas ministradas: o workshop "Processos Digitais de Projeto" (Rhinoceros+Grasshopper) realizado no Curso de Arquitetura e Urbanismo da Universidade Federal de Mato Grosso do Sul (UFMS) em 2015, e o workshop "Processos Digitais de Projeto: Metodologia em Revisão" (Rhinoceros+Grasshopper), realizado junto ao Nó.Lab, no curso de Arquitetura e Urbanismo da Universidade Federal de Viçosa (UFV), também em 2015.

Para o planejamento, tanto da disciplina e sua inclusão no novo projeto pedagógico do Curso de Arquitetura e Urbanismo da Universidade Federal de Mato Grosso do Sul como dos workshops realizados, foi necessário criar uma estrutura no nível do metadesign dasetapas relacionadas ao desenvolvimento de processos baseados em uma lógica de pensamento paramétricos e algorítmicos, que pudesse abordar o problema do projeto arquitetônico a partir de outras considerações. Estas considerações levam em conta algumas teorias, advindas de vários campos disciplinares, como por exemploa Cibersemiótica. Em relação a estes aspectos de natureza teórica, acreditamos que a chave de leitura que permite vislumbrar todo o potencial que pode ser desenvolvido, passa necessariamente pela questão da comunicação, do processamento e da organização da informação presentes nos processos digitais de projeto.

\section{Design Cibersemiótico: processos de concepçãoe produção}

Conforme apontado em Alves (2014), o desenvolvimento teórico que cria o termo em inglês Cybersemiotics (Cybernetics + Semiotics), é proposto na virada do século XX para o século XXI pelo filósofo Dinamarquês Søren Brier. Para Brier (2010), existem algumas semelhanças muito interessantes entre a Cibernética de Segunda Ordem, a Biologia Cognitiva com base na Autopoiésis, e a Semiótica Peirceana. Mas também existem algumas diferenças interessantes que o levaram à conclusão de que são complementares em muitos sentidos.

A Cibernética de Segunda Ordem incluiu o observador nos sistemas observados. Uma Cibernética da Cibernética, como desejava o ciberneticista Heinz von Foerster (FOERSTER, 1974). Sistemas observados poderiam ser classificados como máquinas triviais, onde inputs determinam os outputs. Entretanto, sistemas de observação quando sistemas vivos, nunca serão máquinas triviais. Para a Cibersemiótica, a distinção proporcionada pela segunda ordem é justamente poder conceber sistemas semióticos, os quais dependem das influências do ambiente a partir de sistemas cognitivos. A interpretação e a manipulação dos significados, mais do que input, passa a ser uma característica dos sistemas observados.

Assim, verifica-se que os paradigmas da comunicação e dos significados que se estabelecem a partir dela são fundamentais para que arquitetos e designers compreendam a lógica dos computadores, das relações entre seres humanos e máquinas, da importância da parametrização, da bioinspiração e dos processos generativos da arquitetura digital, assim como da internet e dos atores interligados em redes, que constituem agora parte essencial nos métodos de concepção e de colaboração contemporâneos.

Sabemos que propor um método preciso que contemple todas as possibilidades ligadas à processos digitais de projeto seria uma tarefa extremamente difícil, ou praticamente impossível, e portanto, defendemos a tese de que seja possível propor uma estrutura que abarque grande parte das possibilidades, a partir de um ponto de vista mais geral e menos específico. Partimos inicialmente para a definição de uma estrutura cibersemiótica para processos digitais de projeto (Figura 1):

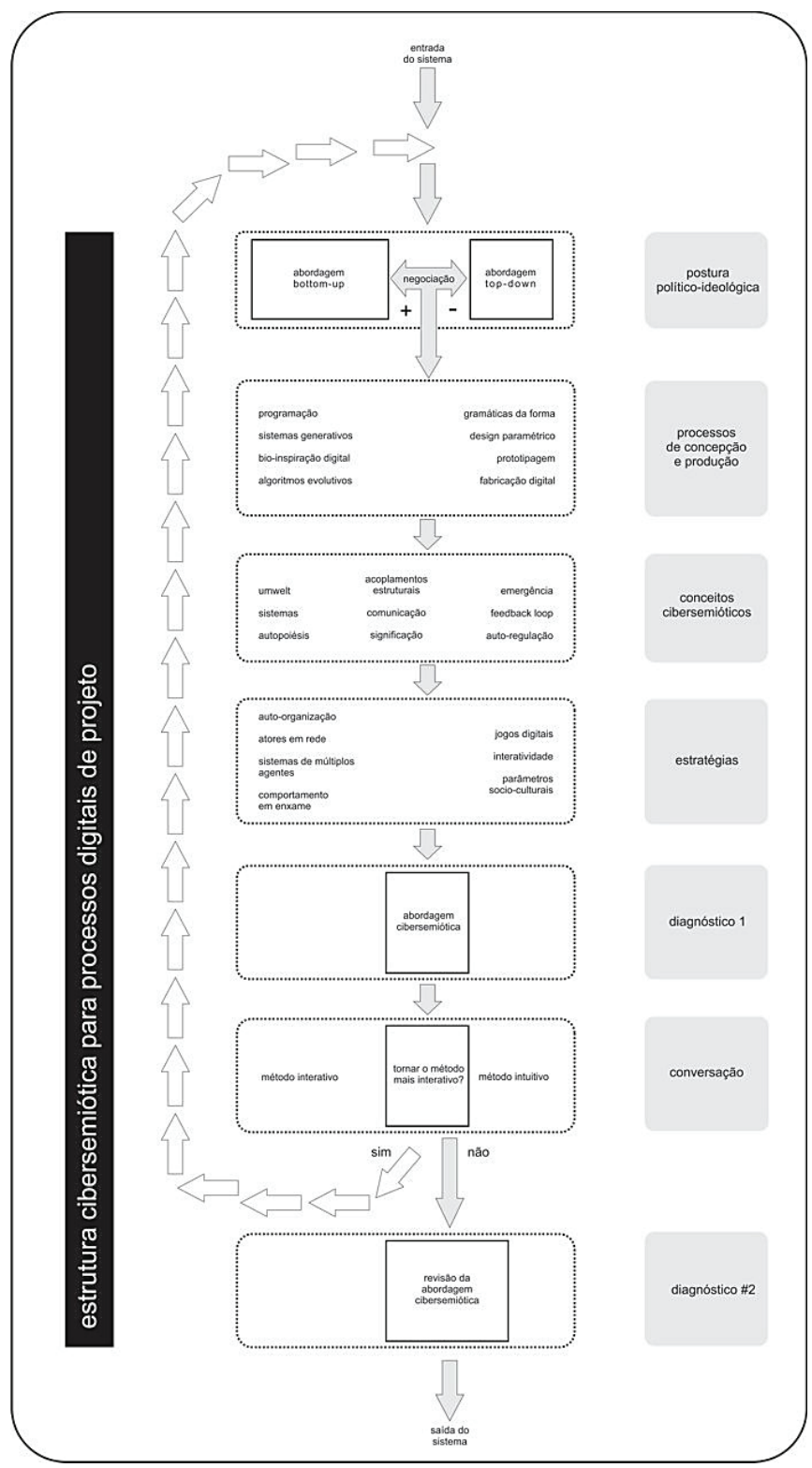

Figura 1: Estrutura Cibersemiótica para Processos Digitais de Projeto Fonte: Alves (2014). 
Mais do que propor um modelo, nossa intenção é discutir possibilidades. Sugerir uma estrutura para processos digitais de projeto que possa oferecer condições mínimas de organização e entendimento sobre as questões envolvidas neste tipo de processo. Porém consideramos fundamental considerar aspectos em aberto, incertezas e níveis de imprevisibilidade em relação às escolhas dos vários atores e os caminhos possíveis em função das particularidades dos sistemas envolvidos.

Andrade et al. (2011), apontam que "existem diversas maneiras de descrever o processo de projeto arquitetônico e como este interfere na produção do edifício", assim como indicam que um projeto arquitetônico é considerado mal estruturado quando os fins e os meios das soluções são desconhecidos e externos ao problema, ou seja, quando a maioria dos problemas de projeto é mal definida.

O diagrama propõe uma estrutura na qual se leve em consideração uma série de abordagens deste a postura político-ideológica de cada projetista ou designer, passando pelos próprios processos de concepção e produção até alguns níveis de verificação a partir da Teoria da Conversação de Gordon Pask e diagnósticos visando o equilíbrio cibernético do sistema.

Por questões de recorte, propomos para o presente artigo o foco na questão específica dos processos de concepção e produção, os quais julgamos passos decisivos e fundamentais para qualquer implementação em nível acadêmico, a partir desse olhar cibersemiótico, conforme detalhado na figura 2:
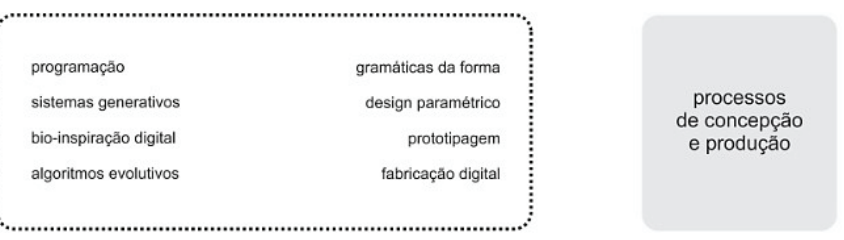

Figura 2: Processos de Concepção e Produção. Fonte: Alves (2014).

O segundo nível de ação proposto dentro do processo de projeto, visa estabelecer um domínio a partir do conhecimento e da aquisição de repertório relacionados aos processos de concepção e produção. Se faz necessário questionar nesta fase se há o domínio de processos em quantidade e/ou qualidade o suficiente para dar conta das demandas e das questões relacionadas à ação anterior. Sabemos que a produção de Arquitetura e Urbanismo a partir da mediação digital reúne uma gama de saberes relacionados às técnicas de programação e à utilização de software e equipamentos digitais. Cabe destacar que o conjunto de saberes e técnicas propostos visa a contextualização dentro de um cenário atual, porém é possível que outros saberes e técnicas sejam incluídos.

Apresentaremos brevemente a seguir a experiência acadêmica realizada na disciplina Representação e Criação
Digital II junto ao Curso de Arquitetura e Urbanismo da Universidade Federal do Mato Grosso do Sul (UFMS).

\section{O Ensino de Processos Digitais de Projeto a partir do olhar cibersemiótico}

As disciplinas de Representação e Criação Digital I e II (http://representacaoeconc.wix.com/informaticaufms) foram propostas e desenvolvidas para a revisão do projeto pedagógico do Curso de Arquitetura e Urbanismo da Universidade Federal de Mato Grosso do Sul, aprovado em 2014 e implementado no ano de 2015.

Essas duas novas disciplinas são reformulações de outras que estavam vigentes até então, Informática Aplicada aos Desenhos de Projetos I e II, a primeira oferecida no $4^{\circ}$ semestre e a segunda no $5^{\circ}$ semestre. Além de trazer as novas disciplinas para o início do curso, entendendo estes saberes como fundamentais para o ingressante, a maior contribuição talvez tenha sido alteração das ementas, as quais anteriormente eram focadas somente na questão da representação arquitetônica. Entendemos processos digitais de projeto como aqueles processos focados na concepção projetual, para além das questões da representação.

A disciplina Representação e Criação Digital II, com carga horária de 68h/a, passou a ocorrer no segundo semestre e possui como pré-requisito a disciplina Representação e Criação Digital I, que ocorre já no primeiro semestre do curso. O seu foco, no entanto, são os processos digitais de projeto, onde as questões relacionadas à concepção digital e à programação são abordadas. De acordo o projeto pedagógico vigente, a ementa consta do seguinte:

Processos Digitais de Projeto. Práticas e experimentos com programas de computador, com foco nas diferentes possibilidades de criação em Arquitetura e Urbanismo. Projetos Paramétricos, Algoritmos Generativos e Morfogênese Digital; Prototipagem e Fabricação Digital; BIM. Cultura digital e Cibercultura; Imersão, interatividade, responsividade e ubiquidade; Cibersemiótica.

Os conteúdos abordados ao longo do semestre letivo iniciam-se com discussões teóricas sobre o pensamento algorítmico e o pensamento paramétrico, apoiados em autores como Oxman (2006), Kotnik (2010), Woodbury (2010) e Oosterhuis (2014). Posteriormente são introduzidas noções de programação apoiados em conceitos cibernéticos, especialmente a partir de Pask (1969) e Dubberly (2009).

Como trata-se de uma disciplina de caráter prático, após estes estudos iniciais o os objetivos da disciplina se concentram em estabelecer um treinamento voltado para a modelagem 3D, utilizando o software Rhinoceros. Vários objetos são modelados com o objetivo de estabelecer um repertório que seja suficiente para proporcionar ao acadêmico a desenvoltura desejada na geração de formas e geometrias complexas (Figura 3): 


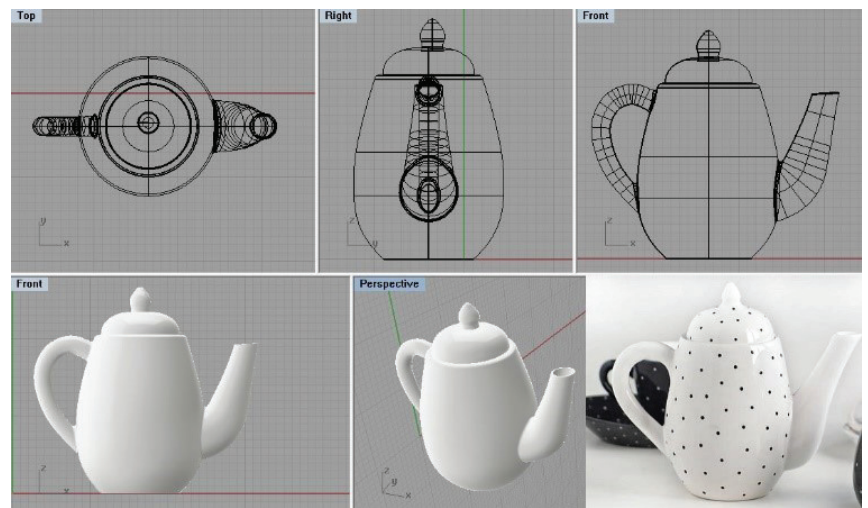

Figura 3: Bule modelado no Rhinoceros a partir do objeto. Fonte: autor (2015).

Passada esta etapa, a disciplina passa-se a concentrar no design paramétrico e na realização de exercícios de programação no Grasshopper, que é um plug-in do Rhinoceros e utiliza-se como referência nesta fase, Woodbury (2010) e Tedeschi (2011).

Os exercícios desenvolvidos passam por algumas etapas de desenvolvimento de acordo com certos níveis de dificuldade. Os primeiros tratam de estabelecer alguns scripts voltados para a compreensão de operações matemáticas e também de conceitos e aplicações como listas, series, grids, range, etc.

Num segundo momento, parte-se para a utilização de scripts que estabelecem algumas relações conceituais com bioinspiração e comportamentos da natureza, como por exemplo o diagrama de Voronoi e os atratores, assim como concepções com estruturas que possam configurar geometrias complexas visando seu posterior processo de fabricação digital, como fatiamentos, waffles e triangulações (Figura 4):

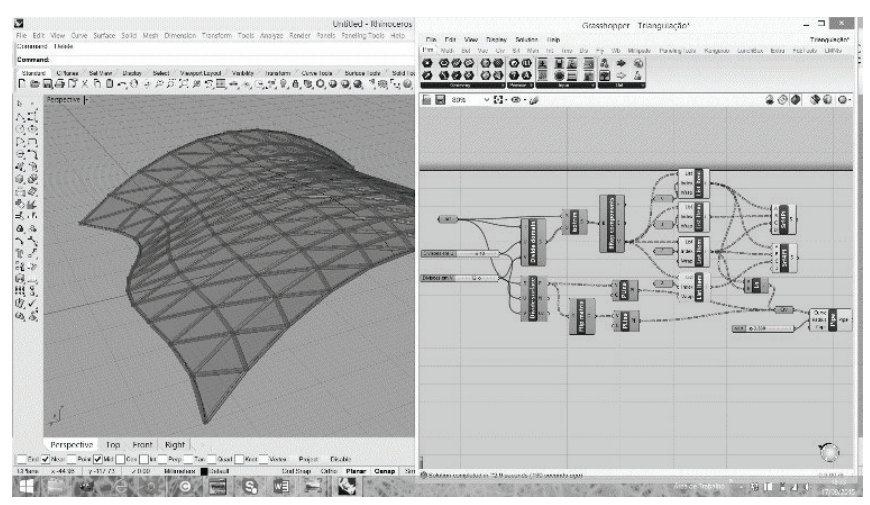

Figura 4: triangulação de uma superfície. Fonte: autor (2015).

Por fim, objetivando a produção de protótipos e introduzir a questão da fabricação digital, trabalha-se alguns scripts para planificação e distribuição em pranchas de corte, no caso da utilização de máquinas do tipo corte à laser ou fresadoras CNC (Figura 5), ou para a geração de arquivos de impressão 3D do tipo STL ou mesmo o G-code para a confecção dos modelos tridimensionais.

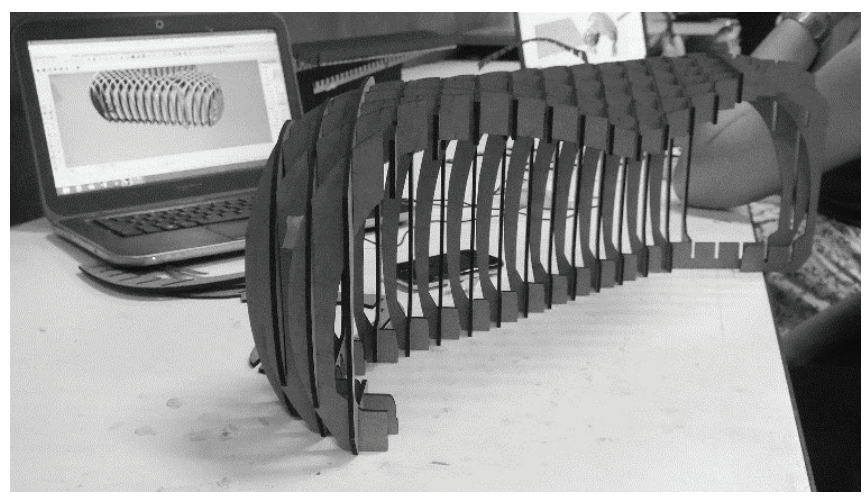

Figura 5: Modelos digital e físico (mdf cortado à laser) de um parklet. Fonte: autor (2014).

Para a produção dos modelos cortados à laser contamos com a parceria do laboratório do Nomads.usp em São Carlos/ SP e eventualmente tem-se recorrido a prestadores de serviço locais, em função do laboratório ainda não estar completamente equipado. Já para a produção de modelos impressos em 3D (Figura 6) está sendo utilizado o laboratório do algo+rtimo em Campo Grande/MS.

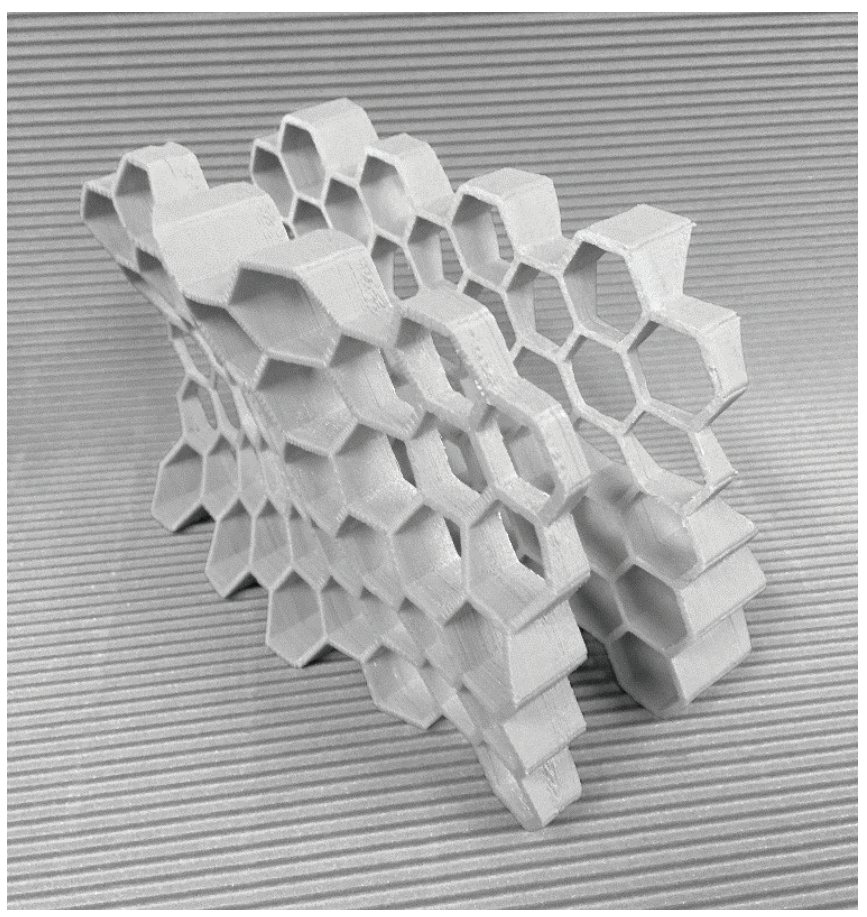

Figura 6: malha alveolar 3D - Impressão em filamento ABS. Fonte: autor (2015).

A condição que as tecnologias de prototipagem e fabricação digital proporcionam traz para a pauta da discussão relacionada ao ensino de projeto em Arquitetura e Urbanismo associado à mediação digital, a questão de que se torna 
urgente o investimento não só em pesquisas e pesquisadores relacionados à área, mas também em equipamentos e laboratórios que viabilizem as experimentações e a retroalimentação entre teoria e prática. Os modelos físicos permitem a evolução do design internamente ao processo de projeto de um modo muito mais dinâmico, retroalimentando o processo o tempo todo, ciberneticamente.

\section{Considerações Finais}

Acreditamos que outras propostas em relação a conceitos advindos de outros campos do conhecimento poderão e deverão ser feitas ainda. Acreditamos também, que a busca cibersemiótica pela colaboração e pela integração transdisciplinar entre diferentes contextos visa contribuir para a formulação de outros saberes científicos, motivando futuras pesquisas para além dos caminhos até o momento percorridos.

Nosso recorte em relação à uma abordagem cibersemiótica para processos digitais de projeto, pretende realizar aproximações, partindo de uma proposta integradora como a Cibersemiótica, porém redirecionada para o campo da Arquitetura e Urbanismo, especificamente ligada a processos de concepção com mediação digital.

Um aspecto decisivo para a adoção da proposta cibersemiótica enquanto desafio cultural, diz respeito à aceitação dos usuários. Não temos certeza de que seja desejável a utilização de uma abordagem cibersemiótica pelos projetistas em sua realidade diária. É provável que algumas ações sejam rejeitadas ou modificadas, enquanto outras poderão ter uma ampla aceitação. Por isso, ao definirmos uma estrutura para processos digitais de projeto, não desejamos impor uma receita estanque e sem capacidade de negociações e ajustes. Pelo contrário, estamos propondo uma possibilidade dinâmica, que se abre a partir da Cibersemiótica e que pode ser adotada por aqueles que se utilizam, e também por aqueles que desejem se aproximar, de processos de projeto com mediação digital. Também nos ambientes acadêmicos, especialmente nas disciplinas que envolvem o ensino de projeto, sabemos que essa discussão está apenas no começo.

\section{Agradecimentos}

Ao Comitê Internacional SIGRADI por fornecer a base para este modelo;
À FAPESP - Fundação de Amparo à Pesquisa do Estado de São Paulo (www.fapesp.br);

À Universidade de São Paulo (USP);

À Universidade Federal de Mato Grosso do Sul (UFMS);

Ao grupo de pesquisa Nomads.usp. (www.nomads.usp.br),

Ao grupo de pesquisa algo+ritmo da UFMS (www. facebook.com/AlgoRitmo.ufms), colegas e alunos que contribuíram e contribuem intensamente para as pesquisas em constante desenvolvimento.

\section{Referências}

ALVES, Gilfranco (20140. Cibersemiótica e Processos de Projeto: Metodologia em Revisão. São Carlos. Tese (doutorado), Instituto de Arquitetura e Urbanismo (IAU), Universidade de São Paulo (USP).

ANDRADE, Max; RUSCHEL, Regina; MOREIRA, Daniel (2011). O Processo e os Métodos. In O Processo de Projeto em Arquitetura: da Teoria à Tecnologia. Kowaltowski et al. São Paulo: Oficina de Textos,

BRIER, Søren (2010). Cybersemiotics: An Evolutionary World View Going Beyond Entropy and Information into the Question of Meaning. Copenhagen: Entropy 12, 1902-1920. DUBBERLY, Hugh; HAQUE, Usman; PANGARO, Paul (2009). What is Interaction? Are there diferente types? ACM Interactions. Volume XVI.1. Modeling Forum.

FOERSTER, Heinz Von (1974). Cybernetics of cybernetics, or the control of control and the communication of communication. Illinois:University of Illinois.

KOTNIK, Toni (2010). Digital Architectural Design as Exploration of Computable Functions. In: International Journal of Architectural Computing. Issue 01, vol. 08.

OOSTERHUIS, Kas (2014). Move That Body: Building Components are Actors in a Complex Adaptive System. pp. 345-360. In: OXMAN, Rivka; OXMAN, Robert. Theories of the Digital Architecture. Abington: Routledge.

OXMAN, Rivka (2006). Theory and design in the first digital age. Haifa: Faculty of Architecture and Town Planning Technion.

PASK, Gordon (1969). The architectural relevance of cybernetics. In: Architectural Design. London.

TEDESCHI, Arturo (2011). Parametric Architecture with Grasshopper. Brienza: Le Penseur.

WOODBURY, Robert (2010). Elements of Parametric Design. New York: Routledge. 